\title{
Analysis of shallow turbulent flows using the Hilbert-Huang transform: a tool for exploring the characteristics of turbulence and coherent flow structures
}

\author{
Kory M. KONSOER ${ }^{1}$ and BRUce RHOADS ${ }^{2}$
}

\begin{abstract}
The Hilbert-Huang transform (HHT) is a method of spectral analysis that is suitable for application to nonstationary and non-linear signals that holds enormous potential for the analysis of turbulent flows in fluvial, aaeolian, and coastal systems. HHT begins with decomposition of the signal into Intrinsic Mode Functions (IMFs) using the Empirical Mode Decomposition method. A Hilbert transform is then applied to each IMF, enabling the calculation of the local spectral characteristics of the signal. Four applications of the HHT are used to demonstrate the utility of this method for spectral analysis of turbulent flows. The method is applied to: (1) velocity measurements of unidirectional flow with high suspended sediment concentration (laboratory), (2) velocity measurements from a combined uni-i-direction and wave flow over a mobile, evolving bed (laboratory), and (3) temperature measurements from the mixing interface of a large river confluence (field). Comparisons among HHT, Fourier, and wavelet analysis are provided, and we identify a number of major benefits of HHT based on these four applications. The results presented show that the spectral method of HHT provides a very useful tool for analysis of turbulence in natural flows and can greatly enhance signal analysis in addition to traditional methods such as Fourier and wavelet analysis.
\end{abstract}

Keywords: turbulence, spectral analysis, Hilbert-Huang Transform, signal processing, river flow

\section{Introduction}

The characteristics of fluid turbulence in rivers, estuaries, coastal environments and the atmosphere are a primary focus of the study of Earth surface processes. Characterization of coherent turbulent structures is of considerable interest because these structures govern the exchange of energy and momentum over a wide range of spatial and temporal scales, thereby influencing sediment transport rates, patterns of erosion and deposition, rates of fluid and constituent mixing, and interactions between flow structure and vegetation (e.g. Niño, Y. and Garcia, M.H. 1996; Buffin-BÉLANGer, T. et al. 2000; ZedLer, E. and STREeT,
R. 2001; Cellino, M. and Lemmin, U. 2004; Wu, F. and YANG, K. 2004; SINGH, A. et al. 2012; Nepf, H. et al. 2013; Venditti, J.G. et al. 2013; Lewis, Q.W. and RhoAds, B.L. 2015). Thus, coherent structures play an important role in the morphologic evolution of fluvial, aeolian, and coastal systems.

Analysis of velocity time series has become an important tool in attempts to identify coherent structures in turbulent flows and to characterize the properties of these structures. Fourier analysis, which yields power spectra illustrating the distribution of turbulent energy associated with different frequencies, is a common method of analysing velocity signals for natural turbulent flows (NikoRA, V.I.

\footnotetext{
${ }^{1}$ Department of Geography and Anthropology, Louisiana State University; Coastal Studies Institute, Louisiana State University, 227 Howe-Russell, Baton Rouge, LA 70803 USA. E-mail: konsoer1@illinois.edu

${ }^{2}$ Departments of Geography and Geographic Information, Science, Geology, and Ven Te Chow Hydrosystems Laboratory, University of Illinois at Urbana-Champaign, 1301 W Green Street, Natural History Building, Second Floor; MC-150, Urbana, IL 61801 USA. E-mail: brhoads@illinois.edu
} 
and Smart, G.M. 1997; UijttewaAl, W.S.L. and Tukker, J. 1998; Sukhodolov, A. and Rhoads, B. 2001; Rhoads, B. and Sukhodolov, A. 2004; SingH, A. et al. 2010). More recently, wavelet analysis, which yields amplitude-frequency-time spectra, has been used to study the time-dependent behaviour of turbulent flows (Chen, J. and Hu, F. 2003; Hardy, R.J. et al. 2009; Wu, X. et al. 2013). Applications of these two methods have greatly enhanced our understanding of turbulence by identifying important frequency scales of velocity fluctuations in different types of turbulent flows and by providing empirical evidence in support of theoretical analyses, such as energy cascade theory (Kolmogorov, A.N. 1941; SukHodolov, A. 1998; SuKhodolov, A. and RHOAds, B. 2001; Rhoads, B. and Sukhodolov, A. 2004).

Despite these successes, Fourier and wavelet analyses are subject to limitations. To be suitable for Fourier analysis signals must be both stationary and linear, while for wavelet analysis signals must be linear (HuANG, N.E. et al. 1998). Moreover, both methods decompose the velocity signal using pre-defined waveforms. In the case of Fourier analysis, signals are decomposed into sine waves. If the fluctuations in a velocity signal are not well described by sine waves, this approach can spread energy over spurious harmonics that do not necessarily reflect real, physical motions of the fluid. Fourier analysis, since it treats sine-wave oscillations over the entire velocity signal, also cannot capture well (if at all) localized fluctuations in a signal produced by turbulent events that occur only for a short duration of the total signal length. Wavelet analysis, by yielding amplitude-frequencytime spectra, can capture events localized in time; however, the waveform used to analyse the signal is pre-defined by the user. Common forms include the Morlet, Gaussian, or Haar wavelets. Because results depend on the type of waveform selected, analysis of a signal using this method is not unique, complicating comparisons among studies.

To address the limitations of Fourier and wavelet analysis, HuANG, N.E. et al. (1998) proposed a new method for spectral analy- sis of nonlinear and non-stationary data. The cornerstone of this method is Empirical Mode Decomposition (EMD), which decomposes a signal into a finite number of modes, termed Intrinsic Mode Functions (IMFs), using the local timescales present in the signal itself. An important attribute of EMD is that the resulting IMFs have physically meaningful Hilbert transforms. The set of Hilbert transforms of the IMFs is used to generate the amplitude-frequency-time distribution of a signal. This distribution may then be integrated over time to yield the power spectrum of frequency. It is therefore possible to investigate the frequency spectrum for the entire signal as well as the time-evolution of the frequency spectrum.

When HuANG, N.E. et al. (1998) first proposed the Hilbert-Huang transform (HHT) method, they used a time series of wind speed to demonstrate its capabilities. Since then, the HilbertHuang method has been used to investigate periodicities in many different types of data, including time series of rainfall, lake temperature, and wind speed (e.g. RAO, A.R. and Hsu, E.C. 2010), stream gage data (e.g. HuANG, Y. et al. 2009; RAO, A.R. and Hsu, E.C. 2010), ground motion during earthquakes (LoH, C. et al. 2000, 2001), and oxygen isotope data from ice cores (Thomas, E.R. et al. 2009). Application of the HHT method to the study of ocean waves has been especially useful because such waves are non-linear, which limits the applicability of Fourier and wavelet analysis (HuANG, N.E. and $\mathrm{Wu}, \mathrm{Z}$. 2008). Additionally, efforts have been made to establish confidence limits for the EMD (HuANG, N.E. et al. 2003) and to determine the nature of noise in the EMD (Flandrin, P. et al. 2004, 2005; Flandrin, P. and Gonçalves, P. 2004; Wu, Z. and Huang, N.E. 2004, 2005). A full summary of previous applications and advances of the HHT method is beyond the scope of this paper; a more complete description can be found in HuANG, N.E. and $\mathrm{Wu}, \mathrm{Z}$. (2008).

Although the HHT method has been successfully applied in a wide variety of fields, applications of the HHT method to turbulent shear flows, such as those that can occur in riv- 
ers, streams, estuaries and coastal zones, are rare and limited in scope (e.g. Sснмiтt, F.G. et al. 2009; KanAni, A. et al. 2010). Given its robust capacity to deal with nonlinear, nonstationary signals, the HHT method has enormous potential to improve our understanding of turbulent shear flows. The purpose of this paper is to illustrate the value of the HHT method for analysing turbulence in water flows through applications including: (1) velocity measurements of unidirectional flow over a flat sand bed below the threshold for sediment motion (laboratory), (2) velocity measurements of unidirectional flow with high suspended sediment concentration (laboratory), (3) velocity measurements from a combined flow (e.g. a flow with both a unidirectional and oscillatory component of velocity) over a mobile, evolving bed (laboratory), and (4) temperature measurements from the shear layer of a large river confluence (field). These examples were specifically chosen to highlight the advantages of the HHT method. The applications show that the method can be used to identify intermittency in an otherwise stationary velocity or temperature signal, analyse time-dependent flows, and capture fluctuations in a signal that are not sinusoidal. EMD also provides a means of noise-removal and signal de-trending. The HHT method is not a replacement for Fourier or wavelet analysis, but it can provide useful additional information on the character of turbulent flows when used in conjunction with those methods.

\section{Methods}

The Hilbert-Huang transform (HHT) determines the local frequency spectrum (i.e. the frequency spectrum at each individual time step) of a signal by applying a Hilbert transform to a complete set of locally orthogonal modes, or intrinsic mode functions (IMFs), of the signal. The HHT method consists of two main steps. First, the original signal is decomposed into a set of IMFs using a method termed Empirical Mode Decomposition (EMD). Next, a Hilbert transform is applied to each IMF and the frequency spectrum of the signal is calculated.

\section{Empirical Mode Decomposition}

The method of EMD is used to transform the signal into a set of IMFs (HuANG, N.E. et al. 1998), which satisfy the following two conditions: (1) the number of extrema (maxima or minima) in the IMF must be equal to the number of zero crossings (e.g. where the signal crosses the x-axis) or differ by one, and (2) the average of the envelope curve of the IMF maxima and the envelope curve of the IMF minima must equal zero at each time step. The HHT method calculates these modes from the signal itself, with no waveform defined a priori. Each IMF represents characteristic oscillations over a narrow range of frequencies, which occur locally, intermittently, or persistently throughout the sample length of the signal. The decomposition procedure involves several steps (for full details of the method, see the original work by HuAng, N.E. et al. 1998):

1. Create upper and lower envelope curves $e_{\max }(t)$ and $e_{\text {min }}(t)$ for the signal by fitting cubic splines to the local maxima and minima in the time series.

2. Average the values of the upper and lower envelope curves at each time to get $m_{1}(t)$ (Eqn. 1).

$$
m_{1}(t)=\frac{e_{\max }(t)+e_{\min }(t)}{2}
$$

3. Subtract $m_{1}(t)$ from the signal $x(t)$ to get $h_{1}(t)$ (Eqn. 2).

$$
h_{1}(t)=x(t)-m_{1}(t)
$$

Check to see if $h_{1}(t)$ satisfies the two conditions to be an IMF. If $h_{1}(t)$ satisfies the requirements, it is $I M F_{1}(t)$. If not, treat $h_{1}(t)$ as the new signal and repeat steps 1-3 for " $j$ " iterations until if $h_{1 j}(t)$ is an IMF. This process is referred to as "sifting." Although satisfying the first condition is absolutely necessary for an IMF, if iterations are carried to an extreme to satisfy 
the second condition, physically meaningful amplitude fluctuations can be obliterated, resulting in a pure frequency modulated signal of constant amplitude (HuANG, N.E. et al. 1998). It is therefore necessary to introduce a stopping criterion for the sifting process. This criterion involves imposing a constraint on the magnitude of the standard deviation (Eqn. 3) between two consecutive "siftings"; in the analyses presented in this paper a value of 0.2 is used (HuANG, N.E. et al. 1998):

$$
S D=\sum_{t=0}^{T}\left[\frac{\left|\left(h_{1(k-1)}(t)-h_{1 k}(t)\right)\right|^{2}}{h_{1(k-1)}^{2}(t)}\right]<0.2
$$

4. Calculate the residual $r_{1}(t)$ between the first IMF and the signal (Eqn. 4).

$$
r_{1}(t)=x(t)-I M F_{1}(t)
$$

The residual $r_{1}(t)$ is now the new signal.

5. Repeat steps $1-4$ using $r_{1}(t)$ to determine $m_{2}(t), h_{2 j}(t)$, and the second intrinsic mode function $I M F_{2}(t)$.

6. Calculate the new residual $r_{2}(t)$ (Eqn. 5).

$$
r_{2}(t)=r_{1}(t)-I M F_{2}(t)
$$

7. Repeat steps $1-7$ to obtain $\mathrm{IMF}_{3^{\prime}} \mathrm{IMF}_{4^{\prime}} \ldots$ $\mathrm{IMF}_{\mathrm{n}}$ until $r_{n}(t)$ becomes a monotonic function or has only one extreme.

The EMD process produces $n$ orthogonal IMFs and a residual $r(t)=r_{n}(t)$. The sum of the $n$ IMFs and the residual $r(t)$ equals the original signal $x(t)$ (Eqn. 6). Performing this summation is a check to determine that the decomposition is complete.

$$
x(t)=\sum_{j=1}^{n} I M F_{j}+r_{n}
$$

During each iteration of the decomposition, progressively lower frequencies of characteristic oscillations remaining in the signal are "sifted" out into a new IMF. The resulting IMFs encompass a narrow range of the frequencies present in the original signal, and as mode number increases, the mean frequency associated with that mode decreases. As with Fourier analysis, the sample length and the Nyquist frequency (half the sampling frequency) set the limit on the lowest and highest frequencies, respectively, that can be identified in the signal. The residual, which must be monotonic or contain only one extreme, represents the trend of the signal over the sample length.

\section{Hilbert transform}

The Hilbert transform is used to extract the local frequency and amplitude from each IMF for spectral analysis. The IMFs, as extracted from EMD, satisfy the two conditions required to define meaningful frequencies using the Hilbert transform. The Hilbert transform is the convolution of a function $f(t)$ with $1 / \pi t$ (Eqn. 7).

$$
\tilde{f}(t)=\frac{1}{\pi} \int_{-\infty}^{+\infty} \frac{f(\tau)}{t-\tau} d \tau
$$

A real function $f(t)$ and its Hilbert transform $f(t)$ form a strong complex analytic signal, $Z(t)$ in which the real $(r)$ part of $Z(t)$ is $f(t)$ and the imaginary (i) part is given by $\tilde{f}(t)$ (Eqn. 8).

$$
Z(t)=f(t)+i \tilde{f}(t)
$$

The Hilbert transform is applied to each of the $n$ IMFs, forming $n$ strong complex analytic signals (Eqn. 9a), which can be also be written in a polar form (Eqn. 9b).

$$
\begin{gathered}
Z_{j}(t)=I M F_{j}(t)+i \overparen{I M F_{j}}(t)=Z_{r j}(t)+i Z_{i j}(t) \\
Z_{j}(t)=A_{j}(t) e^{i \theta(t)}
\end{gathered}
$$

The local amplitude $A_{j}(t)$ is given by Eqn. 10 and the local angular frequency $\omega_{n}$ and frequency $f_{i}(t)$ for each IMF are computed as shown in Eqn. 11a and Eqn. 11b.

$$
\begin{gathered}
A_{j}(t)=\sqrt{Z_{r j}^{2}+Z_{i j}^{2}} \\
\omega_{j}(t)=\frac{d \theta_{j}(t)}{d t} \\
f_{j}(t)=\frac{\omega_{j}(t)}{2 \pi}
\end{gathered}
$$


Thus, there are $n$ values of frequency and amplitude at every time step, which can be expressed as $A_{i}(f, t)$. The amplitude of an IMF at any given time step, for example $A_{3}\left(t_{m}\right)$, reflects the degree to which the corresponding local IMF frequency, $f_{3}\left(t_{j}\right)$, is present in the original signal at that time step, $t_{m}$. The combined set of all $n A_{j}(f, t)$ gives the Hilbert spectrum $H(f, t)$ (Eqn. 12).

$$
H(f, t)=\left\{A_{1}(f, t), A_{2}(f, t) \ldots A_{n}(f, t)\right\}
$$

If two or more IMFs happen to have the same frequency at the same time step, the Hilbert spectrum value for that frequency is the sum of those IMF amplitudes at that time. The Hilbert spectrum can be plotted as discrete points in time-frequency space using a colour scale to represent $H$. Previous studies have applied a weighted Gaussian filter to the discrete points of the Hilbert spectrum to produce a visually continuous plot (HuANG, N.E. et al. 1998).

The plot of the Hilbert spectrum is similar to a wavelet plot in that it displays the time evolution of the frequency spectrum. The main difference between $H(f, t)$ and a wavelet plot is in the method of calculating the amplitude at each frequency-time pair. Wavelet analysis performs a correlation of the signal with a wavelet of varying size at each timestep. The wavelet scale $(a)$ is converted into a "pseudo-frequency" $\left(F_{a}\right)$ using the centre frequency of the wavelet $\left(F_{c}\right)$ and the sampling period of the signal $(\Delta)$ (Eqn. 13).

$$
F_{a}=\frac{F_{c}}{a \triangle}
$$

The form of the wavelet is defined a priori and can influence the results of the analysis substantially. Thus, comparisons between wavelet analyses are limited to those which use the same wavelet. In contrast, the Hilbert-Huang transform does not require any a priori choice of waveform, as the Hilbert spectrum is calculated directly from empirically derived IMFs. The only tuning parameter that affects HHT is the standard deviation tolerance in the shifting process, and, if kept at or below 0.2 (HuANG, N.E. et al. 1998), primarily influences effects the calculation time with negligible changes in the IMFs and residual.

Integrating the Hilbert spectrum over time gives the marginal Hilbert spectrum $h(f)$ (Eqn. 14).

$$
h(f)=\int_{0}^{T} H(f, t) d t
$$

This spectrum is similar to the Fourier spectrum in that it represents the contribution of a given frequency to the original signal. The marginal Hilbert spectrum differs substantially from the Fourier spectrum in that the HHT method can capture periodicities that occur only locally or intermittently in a signal - a capability that Fourier analysis lacks. Thus, peaks in the marginal Hilbert spectrum may represent frequencies that are present locally, intermittently or throughout the whole sample length. In contrast, the Fourier spectrum only captures periodicities that are present persistently over the entire sample length. Peaks in the marginal Hilbert spectrum $h(f)$ can be examined in detail via the Hilbert spectrum $H(f, t)$ to determine if these peaks represent persistent periodicities in the signal or intermittent variations in signal amplitude at certain frequencies. The frequency spectrum of the signal can also be investigated in more detail by integrating each $A_{j}(f, t)$ separately, yielding the marginal spectrum of each IMF. Such an approach is useful for identifying the relative contributions of characteristic oscillatory modes in a signal to the overall spectral characteristics of the signal and for comparing characteristic modes among different signals (Huang, N.E. et al. 1998; Konsoer, K.M. and RhoAds, B.L. 2014). The Hilbert spectrum may also be integrated over frequency to give the "Instantaneous Energy" (IE) at each time step (Eqn. 15). Large values of IE reflect local high amplitude oscillations, a metric of signal energy, of unspecified frequency:

$$
I E(t)=\int_{0}^{f_{\max }} H(f, t) d f
$$

In summary of this method section, HHT provides an alternative approach to analys- 
ing temporal or spatial signals in spectral space compared to Fourier and wWavelet analyses, and offers provides an additional methods of decomposing a the signal and/or integrating it over time or frequency space. Importantly, HHT does not require the selection of a waveform for the spectral analysis a priori. In the next sections, we illustrate using present through various hydrodynamic examples the utility of the HHT method.

\section{Results and discussion}

\section{Application to clay-laden flows}

To illustrate the capabilities of the method to characterize complex turbulent flows with high degrees of intermittency, HHT analysis is applied to velocity data for clay-laden water flows, which exhibit flow characteristics that vary both with the concentration of suspended sediment and the mean velocity. Previous work has identified five types of clay flows based on the effect of increasing clay concentration on the characteristics of fluid turbulence: turbulent flow (TF), turbulenceenhanced transitional flow (TETF), lower and upper transitional plug flow (LTPF/UTPF) and quasi-laminar plug flow (PF) (BAAs, J.H. et al. 2009). HTT analysis was performed on streamwise velocities measured in four types of clay-laden flows (Runs 10-1, 10-19, 10-22 and 10-26 from BAAs, J.H. et al. 2009) (Table 1). These flows have similar depth-averaged velocities and depths, but different clay concentrations. Velocity signals for all four flows were measured $5 \mathrm{~mm}$ above the bed with an ultrasonic Doppler velocity profiler (UDVP) operating at a sampling frequency of 125
$\mathrm{Hz}$ (for details see BAAs, J.H. et al. 2009). The UDVP measures the component of velocity parallel to the instrument beam at 128 points along the beam length.

Velocity time series for each of the four types of flows have distinctive characteristics (BAAs, J.H. et al. 2009) (Figure 1). Signals for the TF and the TETF flows are similar in form, but amplitudes of turbulent fluctuations for the TETF are greater in magnitude than those for the TF. The LTPF exhibits distinct low-frequency oscillations compared to TF and TETF, whereas UTPF is characterized by intermittent "saw-tooth" shaped oscillations (BAAs, J.H. et al. 2009). The Fourier and marginal HHT spectra for each of the four flows are generally similar in form, but the Fourier spectra exhibit more variability than the HHT spectra (Figure 2). The Fourier and Hilbert spectra of the LTPF and UTPF have slightly steeper slopes than spectra for the TF and TETF, but otherwise all four spectra have slopes close to $-5 / 3$ - the value expected for the energy cascade associated with the inertial subrange of turbulent flows (Kolmogorov, A.N. 1941; Sukhodolov, A.N. and UiJTtewaAL, W.S.J. 2010).

By contrast, the full Hilbert spectra of the four flows, plotted as filled contours, differ considerably from one another (Figure 3). The TF Hilbert spectrum and the TETF Hilbert spectrum are similar in that the Hilbert amplitude fluctuates intermittently, with a few localized high-amplitude sections. The TETF Hilbert spectrum exhibits consistently higher Hilbert amplitudes than the TF Hilbert spectrum over the whole range of frequencies and over the entire duration of the signal.

As expected based on the apparent periodicity of the velocity signal, the Hilbert

Table 1. Details for the four clay-laden flows

\begin{tabular}{l|l|c|c|c|c}
\hline Run* & Flow type & $\begin{array}{c}\text { Clay concentration, } \\
\text { vol. } \%\end{array}$ & $\begin{array}{c}\text { Depth-averaged } \\
\text { velocity, cm/s }\end{array}$ & $\begin{array}{c}\text { Mean streamwise } \\
\text { velocity }, \mathrm{cm} / \mathrm{s}\end{array}$ & Flow depth, cm \\
\hline $10-1$ & TF & 0.03 & 140.3 & 97.85 & 14.3 \\
$10-19$ & TETF & 12.48 & 143.3 & 96.12 & 14.0 \\
$10-22$ & LTPF & 14.45 & 140.4 & 75.15 & 14.1 \\
$10-26$ & UTPF & 16.44 & 134.9 & 38.83 & 14.8 \\
\hline
\end{tabular}

*From BAAs, J.H. et al. 2009. ${ }^{* *}$ At $5 \mathrm{~mm}$ above the bed. 


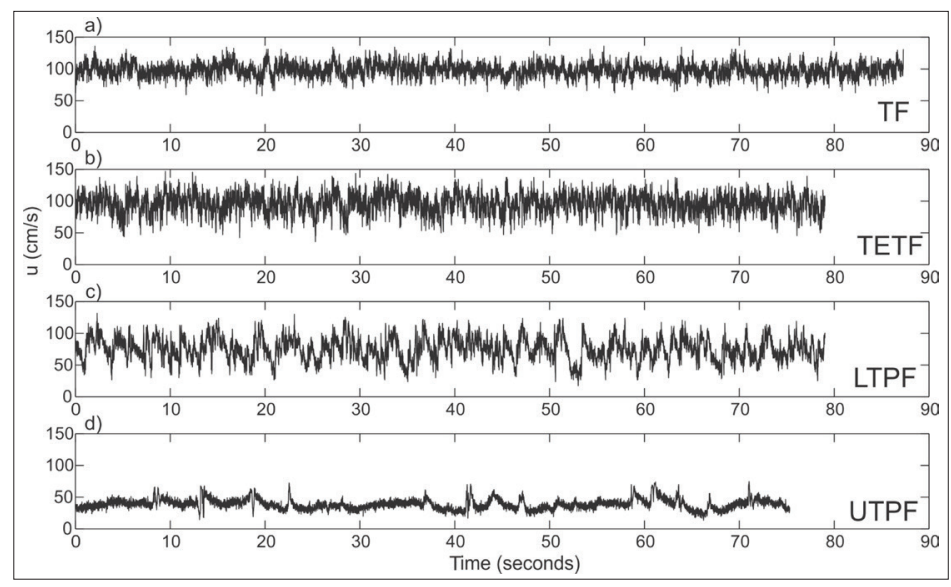

Fig. 1. Time series of streamwise velocity for different concentrations of suspended sediment. Classifications as identified by BAAs, J.H. et al. (2009). - TF = turbulent flow; TETF = turbulence enhanced transitional flow; $\mathrm{LTPF}=$ lower transition plug flow; UTPF = upper transition plug flow

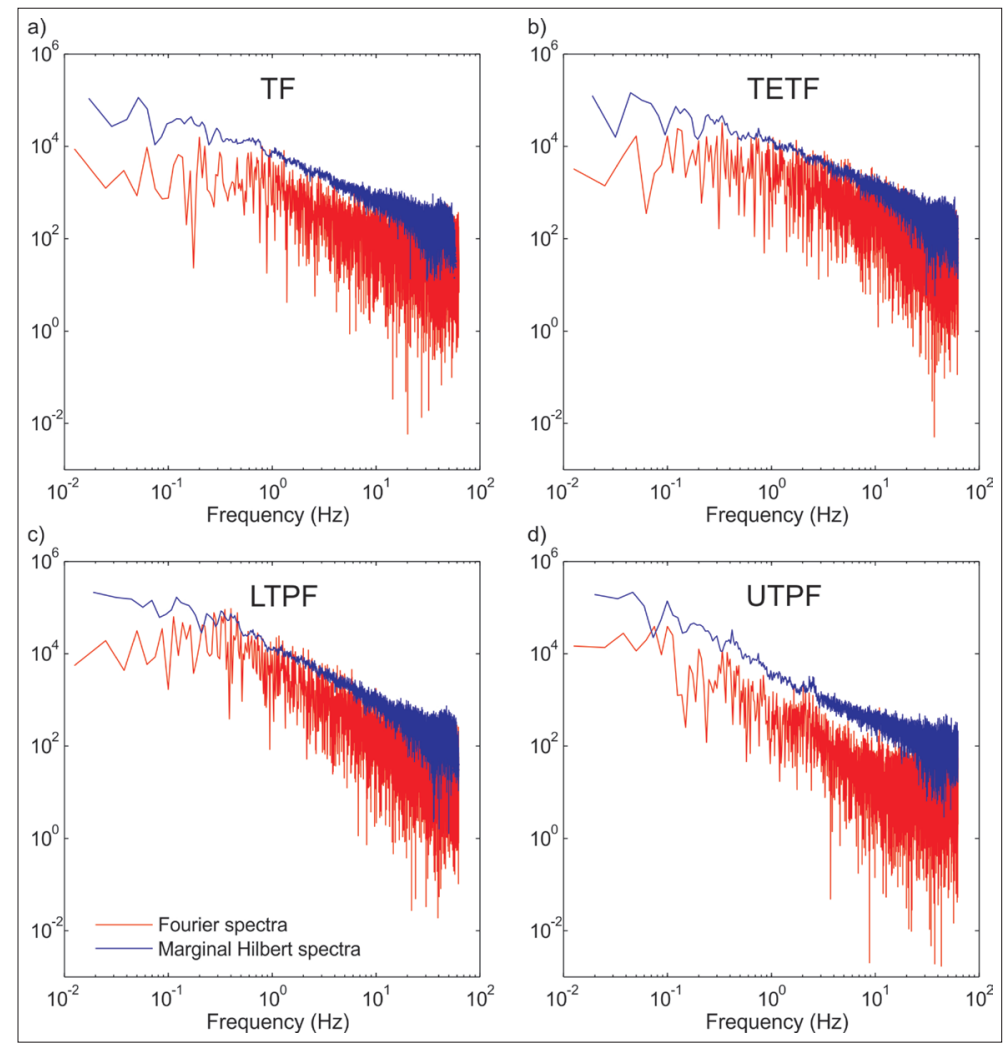

Fig. 2. Comparison of the Fourier spectra to the marginal Hilbert spectra for the four time series of clay-laden flows. - TF, TETF, LTPF and UTPF: For explanation see Fig. 1. 


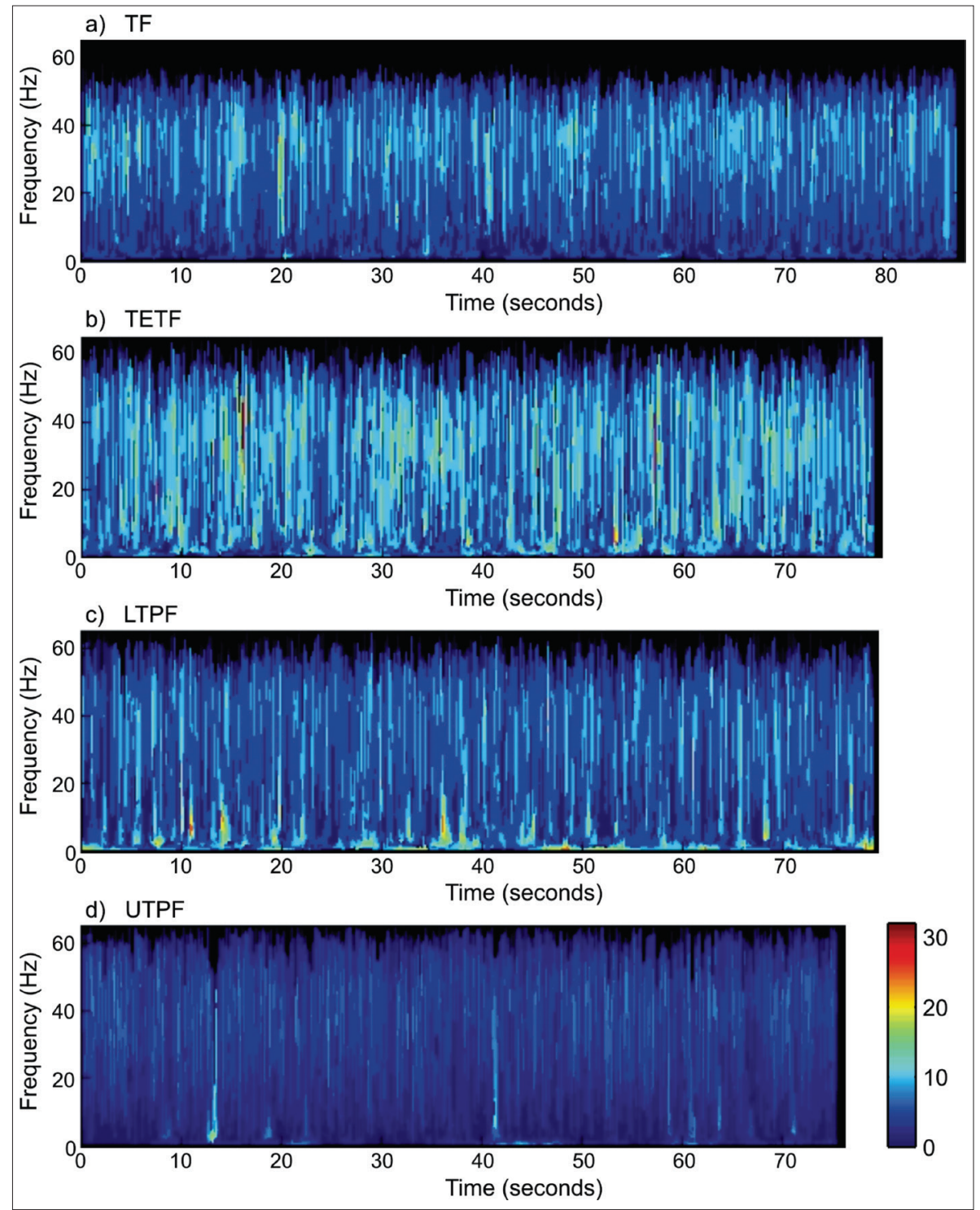

Fig. 3. Full Hilbert spectra for the four time series of clay-laden flows. - TF, TETF, LTPF and UTPF: For explanation see Fig. 1.

spectrum of the LTPF is characterized by intermittent high amplitudes at frequencies less than $\sim 10 \mathrm{~Hz}$. Values of amplitude for these low-frequency velocity variations are the greatest of any of the four flows. Strong low-frequency turbulence for LTPF has been attributed to Kelvin-Helmholz instabilities along an internal basal shear layer that develops between highly turbulent near-bed flow and less turbulent flow in the upper portion of the fluid column (BAAs, J.H. et al. 2009). The Hilbert spectrum of the UTPF is charac- 
terized by small amplitudes at all frequencies, indicating nearly pervasive damping of turbulence by high sediment concentrations. Weak turbulence near the bed, where the vertical gradient in streamwise velocity is largest, produces a number of highly isolated high amplitude fluctuations of the signal at low and intermediate frequencies (e.g. at 13 seconds and $\sim 42$ seconds).

The Instantaneous Energy (IE) plots of the four signals reflect the differences in the Hilbert spectra (Figure 4). Because these plots show the sum of the local Hilbert amplitudes over all frequencies, high values of IE at a particular time may signify a strong local fluctuation of a single frequency or synchronous local fluctuations over multiple frequencies. Averaging the Instantaneous Energy over time gives a measure of the overall oscillatory energy in the signal. The mean Instantaneous Energy of the TETF (mean IE $=493$ ) is higher than that of the TF (mean IE = 221), which is consistent with the higher turbulence intensity of the TETF compared to the TF (BAAs, J.H. et al. 2009). The IE of both the TETF and TF fluctuate over time, reflecting that the strength of the turbulence fluctuates over time. The LTPF Instantaneous Energy consists of systematic low-frequency fluctuations about a relatively high mean value of 690 . These low frequency fluctuations in IE probably reflect Kelvin-Helmholz instabilities within the basal shear layer of the LTPF, which produce high magnitude, low frequency fluctuations in streamwise velocity. The mean Instantaneous Energy of UTPF is the lowest of the four signals (mean $\mathrm{IE}=143$ ); a result that indicates suppression of turbulence related to gelling of the flow (BAAs, J.H. et al. 2009). The IE of the UTPF is also characterized by local spikes, which occur at the same times as the "saw-tooth" velocity fluctuations in the raw velocity signal.

The distinct forms of the Hilbert spectra and Instantaneous Energy plots illustrate nicely the different behaviours of clay-enriched flows described by BAAs, J.H. et al. (2009). Moreover, the full Hilbert spectrum provides a means of distinguishing among different types of clay-laden flows even

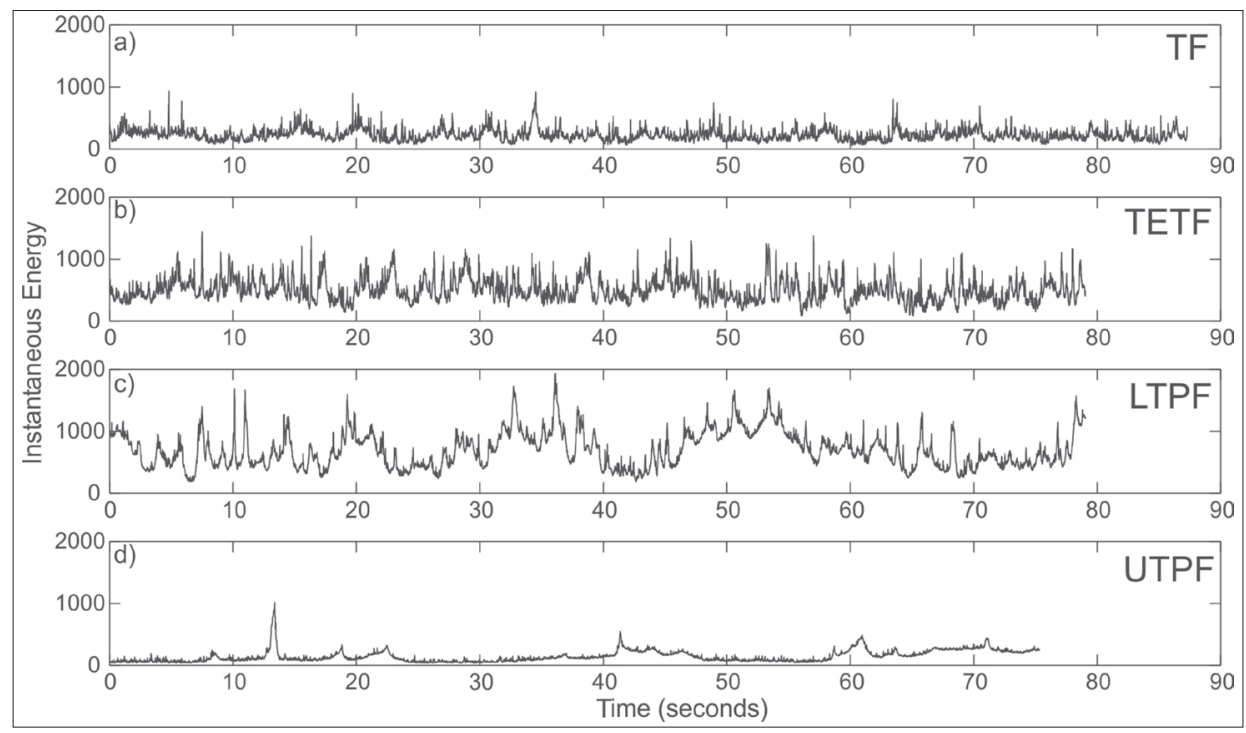

Fig. 4. Instantaneous energy plots for the time series of clay-laden flows. - TF, TETF, LTPF and UTPF: For explanation see Fig. 1. 
though the marginal Hilbert spectra and Fourier spectra of these flows do not differ substantially. Results of the analysis can also be used to characterize differences in the intermittency of these flows, both for individual frequencies and over the entire range of frequencies. The applications of the HHT method to these clay-laden flows shows that it is a robust tool for examining the characteristics of complex turbulent flows, especially when velocity fluctuations of these flows are highly intermittent.

\section{Application to combined flow over a mobile bed}

The second application examines laboratory measurements of non-stationary time series of streamwise and vertical velocity components from a combined flow over an evolving mobile bed. The experiments were conducted in the Large Oscillatory WaterSediment Tunnel facility at the Ven Te Chow Hydrosystems Laboratory at the University of Illinois, Urbana-Champaign (Perillo, M.M. et al. 2014). The combined flow is composed of an oscillatory component with a period of 5 seconds and a maximum oscillatory velocity of $30 \mathrm{~cm} / \mathrm{s}$, and a unidirectional component with mean velocity of $10 \mathrm{~cm} / \mathrm{s}$. The experiment began with a flat, mobile sand bed $\left(D_{50}=250 \mu \mathrm{m}\right)$ and continued for $640 \mathrm{sec}-$ onds through the development of bedforms. Velocities were measured with an ADV at a distance of $1 \mathrm{~cm}$ above the initial flat bed at a sampling frequency of $25.6 \mathrm{~Hz}$. The time series were de-spiked prior to analysis with the HHT method (Goring, D.G. and NikorA, V.I. 2002).

Time series of streamwise $(u)$ and vertical $(w)$ velocities clearly show the non-stationarity of the signal, particularly the variance of the vertical velocities (Figure 5). The marginal Hilbert spectra of the streamwise and vertical velocities both have peaks centred at a frequency of $0.2 \mathrm{~Hz}$, but the peak is much more pronounced for the streamwise velocities (Figure 6). In this case, the peak at $0.2 \mathrm{~Hz}$ is much sharper for the Fourier spectrum than for the marginal Hilbert spectrum because the persistent $0.2 \mathrm{~Hz}$ oscillatory component of the flow over the entire measurement is particularly well suited for Fourier analysis. Overall the marginal Hilbert spectra and the Fourier spectra of the two velocity components are similar in shape (see Figure 6). However, the marginal Hilbert spectrum of streamwise velocity exhibits a broad secondary peak centred on a frequency of $1 \mathrm{~Hz}$, not visible in the Fourier spectrum, suggesting

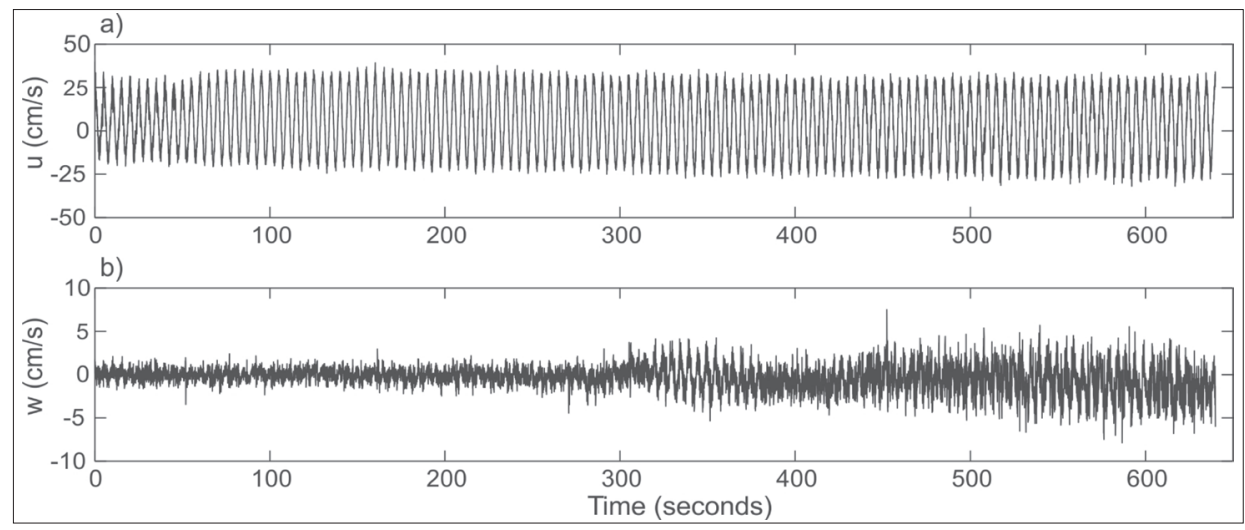

Fig. 5. Time series of (a) streamwise and (b) vertical velocity components of a combined oscillatory and unidirectional flow. (Data courtesy of Perillo, M.M.) 


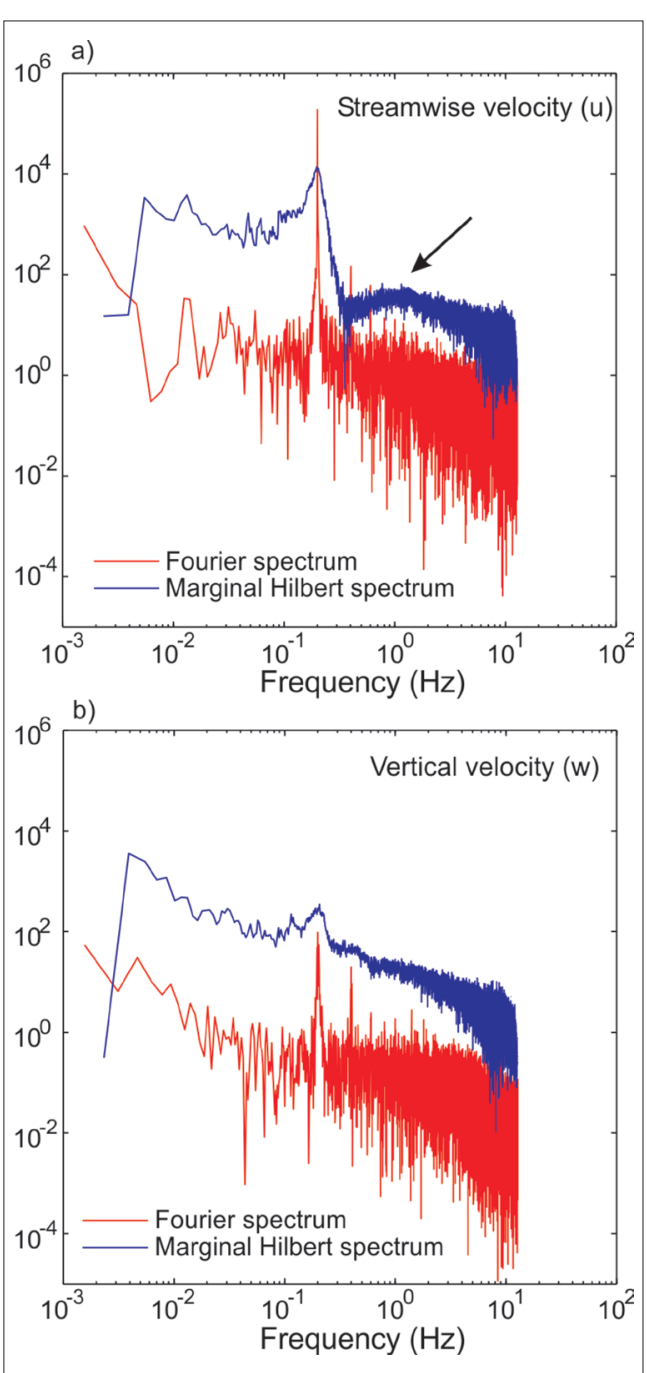

Fig. 6. Comparison of Fourier spectra and marginal Hilbert spectra for (a) streamwise velocity and (b) vertical velocity components for the combined flow described.

that high-frequency turbulent fluctuations are an important part of the signal and that the Fourier spectrum does not capture these high frequencies as well as the marginal Hilbert spectrum (see Figure 6).

A major advantage of the HHT method is its capacity to yield marginal Hilbert spectra for each IMF (Figure 7). The IMFs represent major modes of variability over a narrow range of frequencies within the original time series. For the time series of streamwise velocity, the individual marginal Hilbert spectra of the IMFs show that IMFs 4-6 capture the primary oscillatory component of the combined flow, IMFs 7-11 represent large scale fluctuations not associated with the oscillatory motion, and IMFs 2 and 3, as well as the high-frequency tails of IMFs 4 and 5, represent turbulence associated with high frequency fluctuations (see Figure 7). The first IMF can be associated with measurement noise (Kanani, A. et al. 2010).

The Hilbert spectrum for the streamwise velocity of the combined flow (plotted as discrete points) shows a high-amplitude band centred on $0.2 \mathrm{~Hz}$ (Figure 8, a). By isolating IMFs 2 and 3, which do not include the 0.2 $\mathrm{Hz}$ oscillation, it is possible to examine the time evolution of high frequency turbulent fluctuations independent of the low-frequency oscillatory component of the combined flow (Figure 8, b). To aid interpretation of the time evolution of the signal, the data in the Hilbert spectrum were contoured. After an initial period ( $0-50$ seconds) of increased Hilbert amplitudes associated with turning on the pumps for the experiment, amplitudes diminish as flow over the initially flatbed stabilizes and attains its target oscillatory streamwise velocities. At approximately 290 seconds, the Hilbert amplitudes begin to steadily increase until the end of the time series. This increase in Hilbert amplitude represents an increase in high-frequency turbulent energy associated with the initiation and growth of bedforms. The influence of the initiation of bedforms at roughly 290 seconds is also apparent in the time series of vertical velocity (see Figure 5, b) and its Hilbert spectrum (Figure 8, c). The full Hilbert spectrum for streamwise velocity is in good agreement with the wavelet spectrum for lower frequencies (Figure 8, d).

A comparison of the Hilbert spectrum and wavelet spectrum computed using the Mexican hat waveform illustrates some of the major differences between these two meth- 


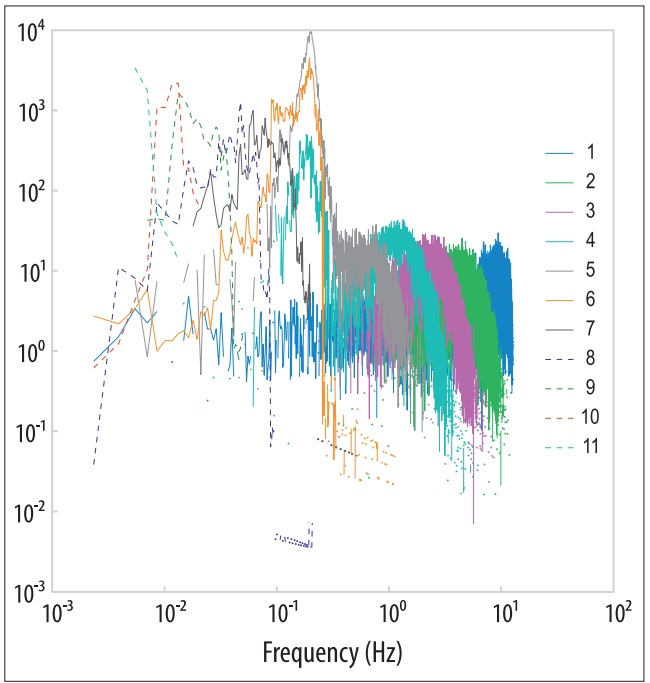

Fig. 7. Marginal Hilbert spectra for each IMF from the streamwise velocity signal of combined flow. Numbers 1-11: For explanation see the text.

ods (see Figure 8). The wavelet spectrum clearly shows an increase in the strength of the $0.2 \mathrm{~Hz}$ oscillation over the first $50 \mathrm{sec}$ onds, as expected from turning on the pumps for the experiment. However, in the wavelet spectrum, the $0.2 \mathrm{~Hz}$ oscillatory component dominates the entire signal and it is difficult to isolate the temporal evolution of the high frequencies. Thus, the ability to isolate individual IMFs is a unique advantage of the HHT method. Moreover, while the Hilbert spectrum provides discrete values of the frequency associated with these high amplitude events (see Figure 8, a), the wavelet spectrum spreads high amplitudes over a range of frequencies (see Figure 8, d).

\section{Detrending and noise-removal with HHT}

Because IMFs represent different oscillatory modes of characteristic frequencies contained within the original signal, the highest and lowest frequency components can be used to de-trend or remove noise from a signal. The HHT method is a robust tool for de-trending because it does not require an initial assumption about the type of trend in the signal and because it can easily remove nonlinear trends. The trend in a signal is simply the residual component from EMD. Subtracting this residual from the signal yields a stationary signal that can be analysed with either the Hilbert transform or the Fourier transform.

A time series of surface water temperatures collected from a mixing interface of a large river confluence of the Wabash and White rivers in Illinois (Figure 9, a-c) is used to demonstrate the de-trending capabilities of HHT (Konsoer, K.M. and RhoAds, B.L. 2014). The data were collected using a boatmounted RDI acoustic Doppler current profiler (ADCP) at a sampling frequency of $1 \mathrm{~Hz}$ over a sampling interval of 117 minutes. The residual trend in the data, as extracted by EMD, shows temperatures increasing nonlinearly throughout the sampling interval (Figure 9, b). This increase in temperature is associated with diel fluctuations of heating. Subtracting the residual from the original time series produces a detrended signal that is stationary over the full length of the time series (Figure 9, c). In this manner, the diel trend in heating is isolated from the fluctuations in water surface temperature that result from the dynamics of the mixing interface at the confluence without assuming a certain type of trend a priori.

Assuming the surface water temperature acts as a passive marker of the fluid from the two tributaries, it is possible to extract information about the mixing interface from the frequency spectrum of the detrended temperature data. The Fourier spectrum of the original signal contains the energy associated with the low frequency diel fluctuation in temperature (Figure 10, a), and is therefore greater than the Fourier spectrum of the detrended signal at frequencies less than $\sim 10^{-2}$. The Fourier spectrum of the detrended signal is nearly identical to the marginal Hilbert spectrum of the original signal, in which the residual trend is automatically excluded from calculations. The power-law form of the frequency spectrum of the detrended signal re- 


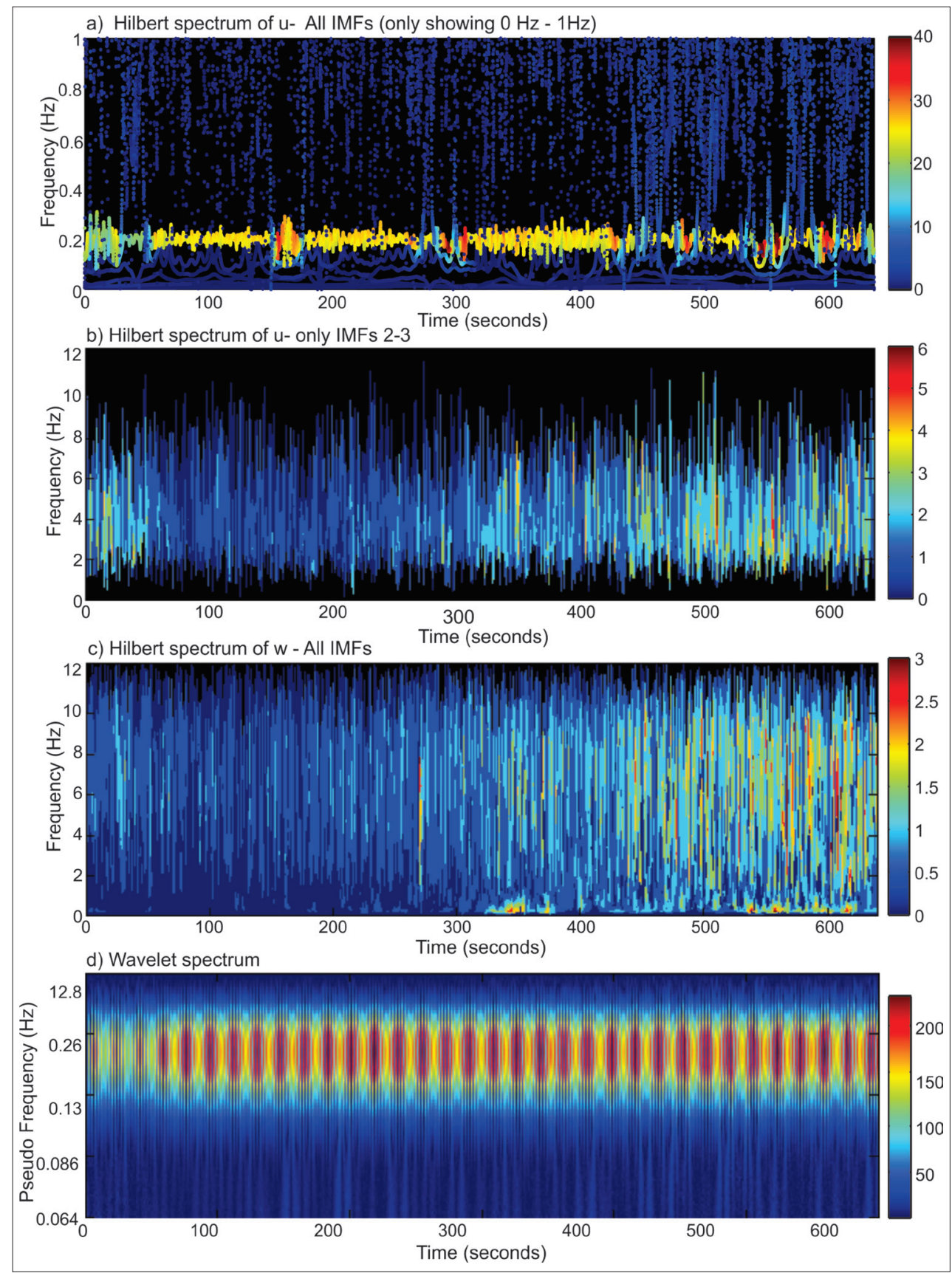

Fig. 8. The full Hilbert spectrum for the streamwise component of the combined flow signal - shown in Figure 5, upper part - plotted as discrete points (a); the Hilbert spectrum for only IMFs 2-3, plotted as filled contours (b); Full Hilbert spectrum for the vertical velocity component of combined flow, plotted as filled contours (c); and wavelet spectrum calculated using the Mexican Hat waveform (d) with scale converted to pseudo-frequency using Eqn. 13. 


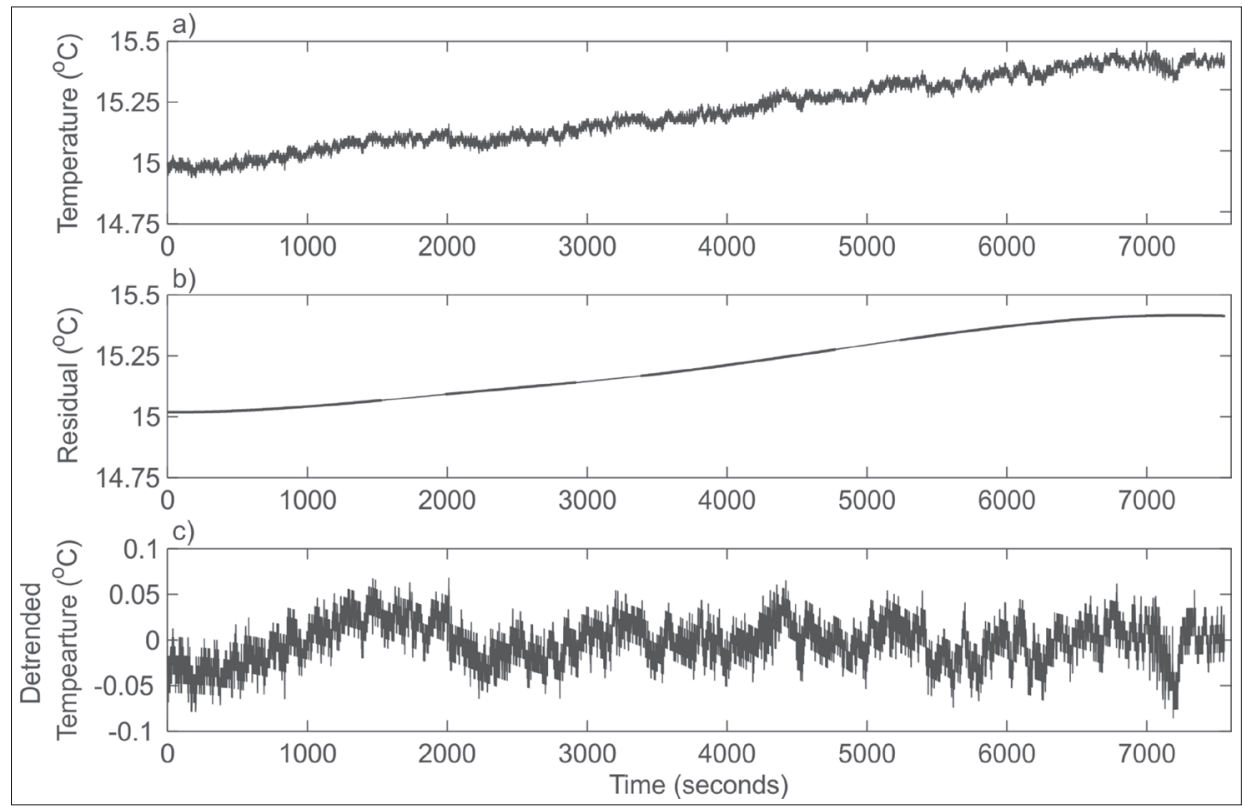

Fig. 9. Surface temperature at a fixed location along a mixing interface at a large river confluence, measured over the course of $\sim 2$ hours (a); Residual of the temperature signal as extracted from the EMD process (b);

Detrended surface temperature, calculated by subtracting the residual from the original signal (c).

flects the variety of scales at which fluid is exchanged across the mixing interface at the confluence. A more detailed interpretation of the flow structures at the Wabash-White confluence is presented in Konsoer, K.M. and RHoAds, B.L. (2014). In addition to detrending hydraulic signals, the HHT method can also be used to de-trend and analyse morphologic data, as was shown for outer bank roughness characteristics along the meandering Wabash River (Konsoer, K.M. et al. 2017).

The first IMF, which represents the highest frequency fluctuations, contains most of the measurement noise in the signal (HuANG, E.N. et al. 2003; KanAni, A. et al. 2010). To remove this noise, the first IMF can be subtracted from the original signal prior to Fourier analysis (Figure 10, b) or the marginal Hilbert spectrum of the first IMF can be excluded from the total marginal Hilbert spectrum (see Figure 10, b). In either case, this adjustment to the data removes the flat, high frequency tail of the spectrum that is dominated by signal noise.

\section{Conclusions}

The examples presented herein demonstrate the unique capabilities of the Hilbert-Huang transform method to shed light on the characteristics of turbulent shear flows. In particular, Hilbert-Huang transform analysis is well-suited for identification of intermittent or localized turbulent events and for analysis of turbulence characteristics over specific frequency ranges. The major benefits of the Hilbert-Huang transform include:

- The Hilbert spectrum is discrete, permitting precise identification of the frequency of a distinct high-amplitude turbulent event occurring at a specific time.

- The Hilbert spectrum allows for the analysis of non-stationary time-evolving flows, enabling exploration of how the spectral characteristics of flow properties vary with changing boundary conditions.

- The Hilbert spectrum is useful in identifying intermittency and determining the character- 
istics of intermittent turbulent events in signals that otherwise appear to be stationary.

- The Hilbert spectrum provides an additional means of distinguishing types of flows that may show similar marginal Hilbert spectra or Fourier spectra (e.g. the four types of clay-laden flows).

- The Hilbert-Huang transform allows for isolation of individual IMFs, enabling detailed analysis of key frequency ranges.

- The EMD process provides a means of detrending and noise-removal that requires no a-priori assumptions about the signal.

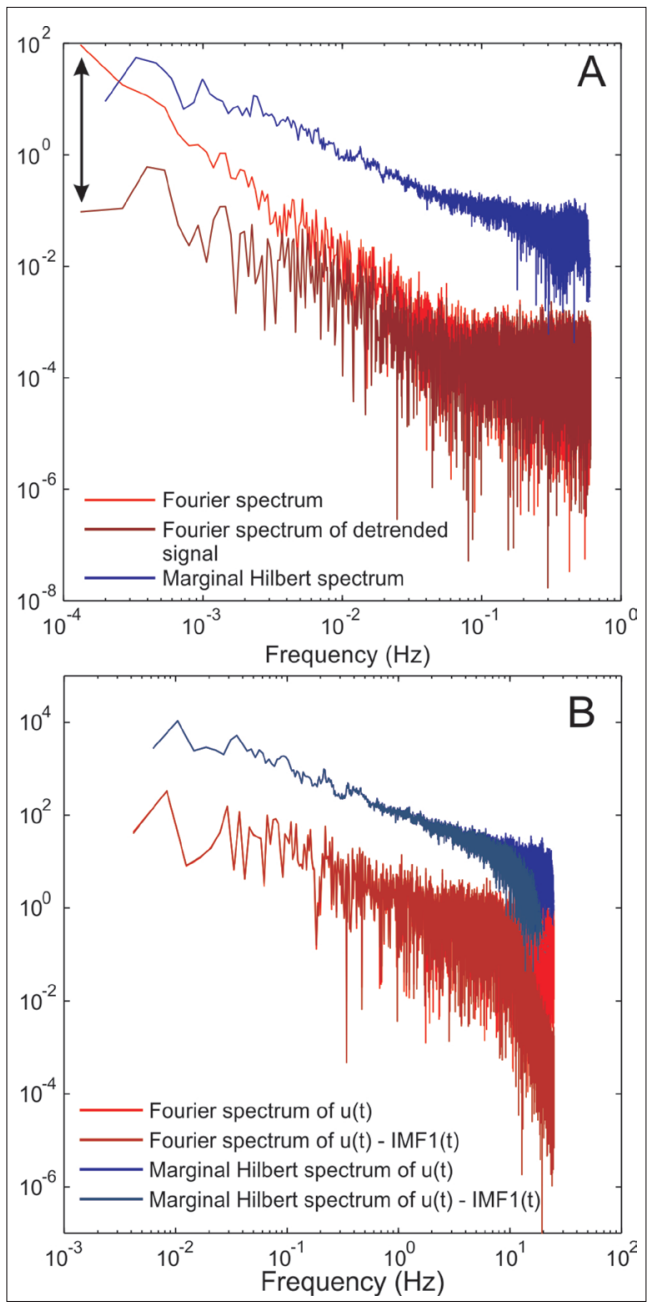

Because of these attributes, Hilbert-Huang transform analysis is useful as an additional tool for spectral analysis of data from these types of flows, but is not a replacement for Fourier or wavelet analysis. Further application of the Hilbert-Huang transform method to turbulent shear flows may provide new insights into how the frequency spectrum of these flows is influenced by strong intermittency, non-stationarity, and non-linearity.

Acknowledgements: The authors would like to acknowledge and thank Jessica LeRoy for her major contributions to the data analysis and writing of this manuscript. This manuscript was improved through thoughtful reviews by the editor and anonymous reviewer. The authors also gratefully thank Mauricio M. Perillo as well as Jaco Bass, Jim Best, Jeff PeAKall and Mi WANG for use of their datasets. Matlab routines for the empirical mode decomposition were provided by Patrick FLANDRIN (http://perso.ens-lyon.fr/ patrick.flandrin/emd.html). Work conducted for this project was supported by NSF Grant BCS-0453316.

Fig. 10. Fourier spectrum of the original temperature signal (red), Fourier spectrum of the detrended temperature signal (dark red) and the marginal Hilbert spectrum of the original temperature signal (blue) - double-arrow line indicates low frequency energy associated with residual trend in Fourier spectrum (a); Examples of noise removal using EMD for the streamwise velocity of the unidirectional flow. The light red line and light blue line show the Fourier spectrum and the marginal Hilbert spectrum, respectively, prior to noise removal and the darker lines show the spectra following noise-removal (b). 


\section{REFERENCES}

Baas, J.H., Best, J.L., Peakall, J. and Wang, M. 2009. A phase diagram for turbulent, transitional, and laminar clay suspension flows. Journal of Sedimentary Research 79. 162-183. Doi:10.2110/jsr.2009.025.

Buffin-Bélanger, T., Roy, A.G. and Kirkbride, A.D. 2000. On large-scale flow structures in a gravel-bed river. Geomorphology 32. (3-4): 417-435. Doi:10.1016/ S0169-555X(99)00106-3.

Cellino, M. and Lemmin, U. 2004. Influence of Coherent Flow Structures on the Dynamics of Suspended Sediment Transport in Open-Channel Flow. Journal of Hydraulic Engineering 130. (11): 1077-1088.

Chen, J. and Hu, F. 2003. Coherent structures detected in atmospheric boundary-layer turbulence using wavelet transforms at Huaihe River basin, China. Boundary Layer Meteorology 107. 429-444.

Flandrin, P., Rilling, G. and Gonçalves, P. 2004. Empirical mode decomposition as a filter bank. IEEE Signal Processing Letters 11. 112-114. Doi:10.1109/ LSP.2003.821662.

Flandrin, P. and Gonçalves, P. 2004. Empirical mode decompositions as data-driven wavelet-like expansions. International Journal of Wavelets, Multiresolution and Information Processing 2. 477-496. Doi:10.1142/ S0219691304000561.

Flandrin, P., Gonçalves, P. and Rilling, G. 2005. EMD equivalent filter banks, from interpretation to applications. In Hilbert-Huang Transform and Its Applications. Eds.: HuAng, N.E. and Shen, S.S.P., Singapore, World Scientific Publishing Co., 57-74.

Goring, D.G. and NiKorA, V.I. 2002. Despiking acoustic Doppler velocimeter data. Journal of Hydraulic Engineering 128. 117-126. Doi:10.1061/(ASCE)07339429(2002)128:1(117).

Hardy, R.J., Best, J.L., Lane, S.N. and Carbonneau, P.E. 2009. Coherent flow structures in a depthlimited flow over a gravel surface: The role of near-bed turbulence and influence of Reynolds number. Journal of Geophysical Research 114. 1-18. Doi:10.1029/2007JF000970.

Huang, N.E., Shen, Z., Long, S.R., Wu, M.C., Shin, H.H., Zheng, Q., Yen, N., Tung, C.C. and Liu, H.H. 1998. The empirical mode decomposition and the Hilbert spectrum for nonlinear and non-stationary time series analysis. Proceedings of the Royal Society A, 454. 903-995. Doi:10.1098/rspa.1998.0193.

Huang, N.E., Wu, M.C., Long, S.R., Shen, S.S.P., Qu, W., Gloersen, P. and FAn, K.L. 2003. A confidence limit for the empirical mode decomposition and Hilbert spectral analysis. Proceedings of the Royal Society A, 459. 2317-2345. Doi:10.1098/rspa.2003.1123.

HuAnG, N.E. and Wu, Z. 2008. A review on HilbertHuang Transform: Method and its applications to geophysical studies. Reviews of Geophysics 46. 1-23. Doi:10.1029/2007RG000228.
Huang, Y., Schmitt, F.G., Lu, Z. and Liu, Y. 2009. Analysis of daily river flow fluctuations using empirical mode decomposition and arbitrary order Hilbert spectral analysis. Journal of Hydrology 373. 103-111. Doi:10.1016/j.jhydrol.2009.04.015.

Kanani, A., Ahmari, H. and Ferreira da Silva, A.M. 2010. Investigation of horizontal coherent structures in a shallow open-channel flow using velocity signal decomposition. In Proceedings from the International Conference on Fluvial Hydraulics River Flow. September 8-10, 2010. Braunschweig, Germany, 1059-1066.

Kolmogorov, A.N. 1941. Dissipation of energy in locally isotropic turbulence. Doklady Akademii Nauk SSSR 32. 19-21. (in Russian)

Konsoer, K.M. and RHoAds, B.L. 2014. Spatial-temporal structure of mixing interface turbulence at two large river confluences. Environmental Fluid Mechanics 14. 1043-1070. Doi:10.1007/s10652-013-9304-5.

Konsoer, K.M., Rhoads, B., Best, J., Langendoen, E., Ursic, M., Abad, J. and Garcia, M. 2017. Length scales and statistical characteristics of outer bank roughness for large elongate meander bends: The influence of bank material properties, floodplain vegetation and flow inundation. Earth Surface Processes and Landforms 42. 2024-2037.

Lewis, Q.W. and RHOADs, B.L. 2015. Rates and patterns of thermal mixing at a small stream confluence under variable incoming flow conditions. Hydrological Processes 29. (20): 4442-4456.

Loh, C., Lin, C. and Huang, C. 2000. Time domain identification of frames under earthquake loadings. Journal of Engineering Mechanics 126. 693-703. Doi:10.1061/(ASCE)0733-9399(2000)126:7(693).

LoH, C., Wu, T. and HuANG, N.E. 2001. Application of the empirical mode decomposition-Hilbert spectrum method to identify near-fault ground-motion characteristics and structural responses. Bulletin of the Seismological Society of America 91. 1339-1357. Doi:10.1785/0120000715.

Nepf, H., Rominger, J. and Zong, L. 2013. Coherent Flow Structures in Vegetated Channels. In Coherent Flow Structures at Earth's Surface. Eds.: VenditTI, J.G., Best, J.L., Church, M. and Hardy, R.J., Chichester, UK, John Wiley \& Sons Ltd. Doi: 10.1002/9781118527221.ch9.

Nikora, V.I. and Smart, G.M. 1997. Turbulence characteristics of New Zealand gravel-bed rivers. Journal of Hydraulic Engineering 123. 764-773.

Niño, Y. and Garcia, M.H. 1996. Experiments on particle - turbulence interactions in the near-wall region of an open channel flow: implications for sediment transport. Journal of Fluid Mechanics 326. 285-319. Doi:10.1017/S0022112096008324.

Perillo, M.M., Best, J.L. and Garcia, M.H. 2014. A unified model for bedform development and equilibrium under unidirectional, oscillatory and combined-flows. Sedimentology 61. (7): 2063-2085. 
RAO, A.R. and Hsu, E.C. 2010. Hilbert-Huang Transform Analysis of Hydrological and Environmental Time Series. Dordrecht, Springer.

Rhonds, B. and Sukhodolov, A. 2004. Spatial and temporal structure of shear layer turbulence at a stream confluence. Water Resources Research 40. 1-13. Doi:10.1029/2004WR002811.

Schmitt, F.G., Huang, Y., Lu, Z., Liu, Y. and Fernandez, N. 2009. Analysis of velocity fluctuations and their intermittency properties in the surf zone using empirical mode decomposition. Journal of Marine Systems 77. 473-481. Doi:10.1016/j. jmarsys.2008.11.012.

Singh, A., Porte-Agel, F. and Foufoula-Georgiou, E. 2010. On the influence of gravel bed dynamics on velocity power spectra. Water Resources Research 46. 1-10, Doi:10.1029/2009WR008190.

Singh, A., Foufoula-Georgiou, E., Porte-Agel, F. and Wilcock, P.R. 2012. Coupled dynamics of the co-evolution of bed topography, flow turbulence and sediment transport in an experimental flume. Journal of Geophysical Research 117. F04016. Doi:10.1029/2011JF002323.

SukHodolov, A. 1998. Turbulence structure in a river reach with sand bed. Water Resources Research 34. 1317-1334. Doi:10.1029/98WR00269.

Sukhodolov, A. and Rhoads, B. 2001. Field investigation of three-dimensional flow structure at stream confluences. Water Resources Research 37. 2411-2424.

Sukhodolov, A.N. and UijttewaAl, W.S.J. 2010. Assessment of a river reach for environmental fluid dynamics studies. Journal of Hydraulic Engineering 136. 880-888. Doi:10.1061/(ASCE) HY.1943-7900.0000267.

Thomas, E.R., Dennis, P.R., Bracegirdle, T.J. and FrANZKe, C. 2009. Ice core evidence for significant 100-year regional warming on the Antarctic Peninsula Geophysical Research Letters 36. 1-5. Doi:10.1029/2009GL040104.
UijttewaAl, W.S.L. and Tukker, J. 1998. Development of quasi two-dimensional structures in a shallow free-surface mixing layer. Experiments in Fluids 24. 192-200.

Venditti, J.G., Hardy, R.J., Church, M. and Best, J.L. 2013. What is a Coherent Flow Structure in Geophysical Flow? In Coherent Flow Structures at Earth's Surface. Eds.: Venditti, J.G., Best, J.L., Church, M. and Hardy, R.J., Chichester, UK, John Wiley \& Sons Ltd. Doi:10.1002/9781118527221.ch1

WU, F. and YANG, K. 2004. Entrainment Probabilities of Mixed-Size Sediment Incorporating Near-Bed Coherent Flow Structures. Journal of Hydraulic Engineering 130. (12): 1187-1197.

Wu, X., YU, B. and WANG, Y. 2013. Wavelet analysis on turbulent structure in drag-reducing channel flow based on direct numerical simulation. Advances in Mechanical Engineering 5. (1): 1-11. Doi:10.1155/2013/514325.

Wu, Z. and HuAnG, N.E. 2004. A study of the characteristics of white noise using the empirical mode decomposition method. Proceedings of the Royal Society A, 460. 1597-1611. Doi:10.1098/rspa.2003.1221.

Wu, Z. and HuANG, N.E. 2005. Statistical significance test of intrinsic mode functions. In Hilbert-Huang Transform and Its Applications. Eds.: HuAng, N.E. and Shen, S.S.P., Singapore, World Scientific Publishing Co., 107-127.

Zedler, E. and Street, R. 2001. Large-Eddy Simulation of Sediment Transport: Currents over Ripples. Journal of Hydraulic Engineering 127. (6): 444-452. 
